\title{
Clustering Protocol Schemes for Vehicular Ad Hoc Network Environments: A Review
}

\author{
Rajeev Kumar Patial" and Nongmaithem Island Devi
}

Department of Electronics and Communication, Lovely Professional University, Phagwara - 144411, Punjab, India; rajeev.kumar@|pu.co.in, island_devi@yahoo.co.in

\begin{abstract}
Vehicular Ad-Hoc Networks (VANETs) has come up in the late years to improve street and vehicle security, solace to drivers and travelers furthermore the simplicity to activity proficiency. VANET is an uncommon sort of Mobile specially appointed system (MANET) where vehicles are utilized to make portable system. It is the future transportation framework as it gives portable system correspondence among the vehicles. Since the messages in vehicles are communicated at remote medium the VANET correspondence is at high hazard. So to defeat this test group development is proposed. The groups are separated into portability and non versatility in view of their calculation. Review of grouping calculation has been centered around this paper.
\end{abstract}

Keywords: CH (Cluster Head), CHL (Cluster Head Level), GPS (Global Positioning System), Vehicular Specially Appointed Networks (VANET)

\section{Introduction}

The social effects and the compelling monetary effects of vehicle mischance have moved the advancement of Intelligent Transportation Systems (ITS) and exploration. Savvy transportation frameworks primary points are to enhance effectiveness of surface transportation frameworks, wellbeing and security through the wise advances change. These shrewd framework involves diverse sorts of advancements. However most applications uses key as indicated by the capacity of interchanges in the vehicular environment. Vehicular correspondence constitutes of two sorts. They are vehicle-to-vehicle $(\mathrm{V} 2 \mathrm{~V})$ correspondence or vehicleto-roadside (V2R) correspondence. Vehicular specially appointed system (VANET) is the development of correspondence amongst vehicles.

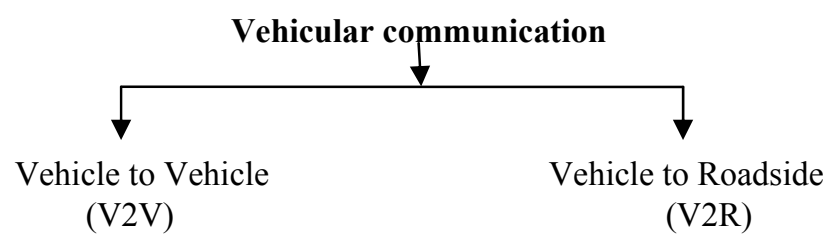

Figure 1. Types of vehicular communication.

This innovation goal here is the vehicles have the ability to speak with each other, see hazards and also react more rapidly than the human reflexes permit. VANET innovation maintains a strategic distance from mishaps on street up to significant degree and if mischance's happen in that case the vehicle crashes survivable. The VANET forestall $80 \%$ mishaps that generally are not brought by inebriated mechanical or drivers disappointments. 
Item advancement gets to be basic because of standard. Models disentangle the item advancement, helps in cost lessening, and empower clients to likewise look at contending items. Interconnectivity and interoperability is accomplished just by utilizing the benchmarks and to empower quick usage of check rise of new item and new innovation ought to be finished.

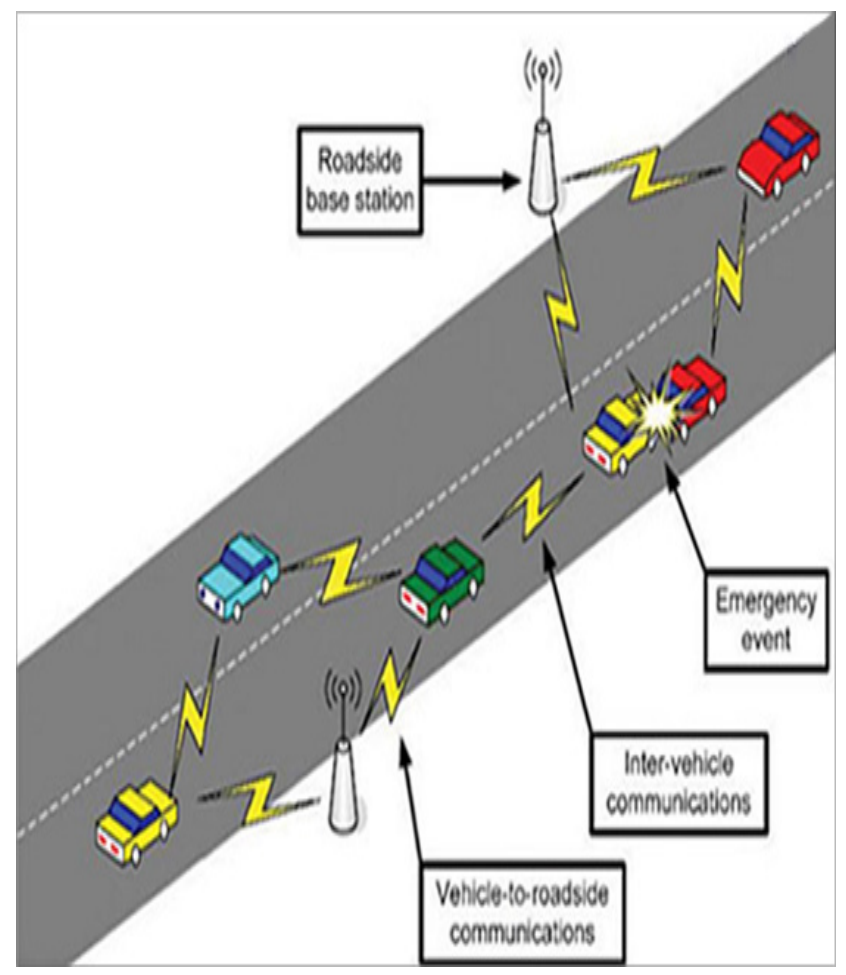

Figure 2. Overview of VANET.

\section{Clustering Protocols for Vanet}

Grouping plans for VANET has been proposed by specialists everywhere throughout the globe. These methods concentrate on different points and uses grouping plans to proposed for MANET for the development of bunch. In this manner, we endeavor to characterize the plans as indicated by the parameters utilized as a part of their particular method. Grouping plan arrangement in vehicular systems is abridged underneath.

\subsection{Mobility based Clustering Schemes}

In this class consider the convention with versatility normal for vehicles as a parameters selection for the groups and bunch heads in given system. Alternate qualities incorporates position, speed, direction, and so forth. The portability grouping procedures is further ordered into two distinct sorts relying upon the bearing of the vehicles on street. It is the non-course based bunching plans and bearing grouping plans.

Table 1. Mobility based on clustering schemes

\begin{tabular}{|l|l|}
\hline \multirow{2}{*}{$\begin{array}{l}\text { Direction based on } \\
\text { clustering schemes }\end{array}$} & $\begin{array}{l}\text { Lane based on clustering schemes } \\
-[2],[3]\end{array}$ \\
\cline { 2 - 2 } & $\begin{array}{l}\text { Vehicle based clustering schemes - } \\
{[1],[4],[5],[6]}\end{array}$ \\
\hline $\begin{array}{l}\text { Non-direction based } \\
\text { clustering schemes }\end{array}$ & {$[7],[8],[9],[10]$} \\
\hline
\end{tabular}

\subsection{Direction based Clustering Schemes}

The course construct grouping plans is center in light of bearing of vehicle for selecting the powerful bunches for vehicular system. Some of these plans likewise concentrate on bearing of the vehicle or path for selecting the bunches or group sets out toward separate system. So as indicated by the distinctions, the bearing based bunching plans can be further ordered into two sorts which are path based grouping plans and vehicle based bunching plans.

\subsubsection{Lane based Clustering Schemes}

The path based bunching's plan considers the heading of activity on street as one of its parameters for ascertaining the effective furthermore nearly stable groups in VANET. The steady bunching plan favorable circumstances diminish the overhead re-grouping and results in a proficient system of topology. The changes in group head occur and in addition bunch reconfigurations dodged cannot differ in VANET. This effect the system strength. There must be few group head change for more steady bunches in the system. To acquire less number of bunch head change, the group part ought to choose hub which is among the bunch individuals that can meet every one of the prerequisites of being a group head for moderately drawn out stretch of time contrasted with rest.

Mohammad S. Almalag et al. $\underline{2}$ propsed a strategy in which the bunch head is chosen by relying upon path with most extreme movement stream. The vehicles know about the street activity on path and they communicate this specific data to adjacent vehicles which helps in deciding the proficiency of bunch head. Every one of the vehicles in system process and also communicate to 
Cluster Head Level likewise called CHL or speed and so on. CHL ascertation is by path normal separation level, path weight, normal speed level of the movement and system availability level.

A vehicle communicates the computed CHL by utilizing intermittent reference points and general movement information in the systems. The vehicle having the most noteworthy CHL worth is chosen as the group head. In like clockwork this procedure of group arrangement is rehashed.

N. Maslekar et al. $\frac{3}{3}$ talked about the plan which go for more stable group and also correct thickness estimation for bunch. The arrangement of group happens even before the street convergence and utilizations the anticipated voyaging way for the bunch development.

By utilizing Global Positioning System (GPS), the vehicle area is known by the system yet course data crossing point is ascertained earlier. The development of bunches begins close to the convergence and relies on upon the course taken by vehicle in the wake of intersection crossing point. Vehicle overwhelming influences the steadiness in groups system.. A notice of overwhelming is sent to the $\mathrm{CH}$ at whatever point a vehicle needs to surpass and on accepting it checks if vehicle can be another $\mathrm{CH}$.

\subsubsection{Vehicle based Clustering Schemes}

The conventions which utilizes the bearing of vehicles for the choice of group heads and bunches.

APROVE ${ }^{6}$ proposed a bunching calculation which underscores on development of stable groups. Here every hub transmit data to every single neighboring hub and every hub autonomously takes choice on bunching. It effectively diminishes the portability and separation between bunch head and part hubs.

$\mathrm{AMACAD}^{5}$ represented the vehicles behaviour and also their mobility group's patterns to increase the cluster lifetime of vehicular network and also global overhead reduction. The protocol increases the lifetime of cluster, manages the mobility, decreases the change in cluster head, group re-clustering and decreases the cluster reaffiliations.

LORA-DCBF ${ }^{4}$ utilizes both GPS and vehicle course for group development. The steering of parcels is done in bounce to jump and hub utilizes the late area data of neighboring hubs. In this manner the parcel misfortune is maintain by the framework.
VWCA $^{1}$ gives the grouping calculation utilizing complex metric structure stable bunches furthermore expanded availability in system. The metric incorporates the course of vehicles, number of neighbors, the doubt esteem parameters and the entropy. Availability and soundness can be upgraded furthermore overhead is diminished by considering parameters for bunch arrangement in system. Vehicle with least weighted value of clusters neighborhood is selected to be cluster head.

\subsection{Non-Direction based Schemes}

Protocols which uses metric for selection of the clusters and cluster heads mobility without direction are classified as non-direction based schemes.

$\mathrm{VMaSc}^{7}$ proposed clustering schemes by selecting cluster head and considering the least mobile function. Least mobility node identification is done by the use of speed difference between network of neighboring nodes. Here the key metric is an relative average speed and clustering depends on change in relative's vehicle mobility of calculating average relative rate of every neighboring hub moving in same heading. In the event that group head doesn't get any parcel from associated part in predefined measure of time then it accepts that the part vehicles are lost. Consequently, the associations between head of pair part are weaker than multi bounce bunching.

ASPIRE $^{10}$ plan is proposed for VANET where grouping is done in appropriated way. It helps in making huge groups and to give high system availability. It decreases the expense of framework in system by utilizing unimportant vehicles on streets.

KCLS protocol ${ }^{8}$ spoken to an area administration convention that consolidates both availability and versatility of vehicles in the system. Bunches can be chosen with tradeoff between correspondences overhead and vehicle area. It expands the group head life time upto 50 percent whwn contrasted with other bunching plans.

To get steady groups the hub with high versatility is contrasted with neighboring hubs and can't be chosen as a bunch head. In the event that chose as group head then the likelihood of bunch breaking and re-grouping is high. In this way, $\mathrm{MOBIC}^{9}$ is propose in which a hub with slightest portable nature is contrasted with neighboring hubs and that ought to be chosen as the bunch head. 


\subsection{Non-Mobility based Schemes}

Non-portability based plans are VANET plans which use bunching strategy yet does not utilize versatility as one its measurements. The few of the non-portability based plans are given underneath:

Keisei Okano et al..$^{11}$ speaks to an independent grouping plan that chooses system door hub progressively as indicated by the system topology changes in heterogeneous versatile impromptu system. At whatever point a system passage hubs is to be chosen the group head takes choice on whether to choose or to sit tight for a particular measure of time. To evade wavering in two adjoining bunches, the holding up periods is computed on contrasting its own particular system address and the system location of the neighboring systems.

Slawomir Kuklinski et al. $\stackrel{12}{\text { grouping calculation is }}$ talked about to frame stable bunches and long living for solid correspondence. It is a bunching plan with complex metric, capacity of thickness of association diagram, join quality and movement condition. in this strategy bunch arrangement depends on elements like availability level, join quality, hub notoriety and so forth.

A CBLR (Cluster based area steering) is proposed by

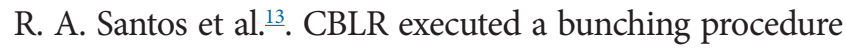
assessing the between vehicular movement information on a motorway utilizing multi-bounce system. Hubs in vehicular system utilize HELLO messages to convey the state. New hub which enters the systems can join a current group or make new bunch by going about as a group heads. It is expected that every one of the hubs know position and area of different hubs in a group. In this the group head keep up a bunch table which contain the locations and geographic areas of member nodes. A group neighbors table is kept up by the bunch head that contains data about neighboring bunch.

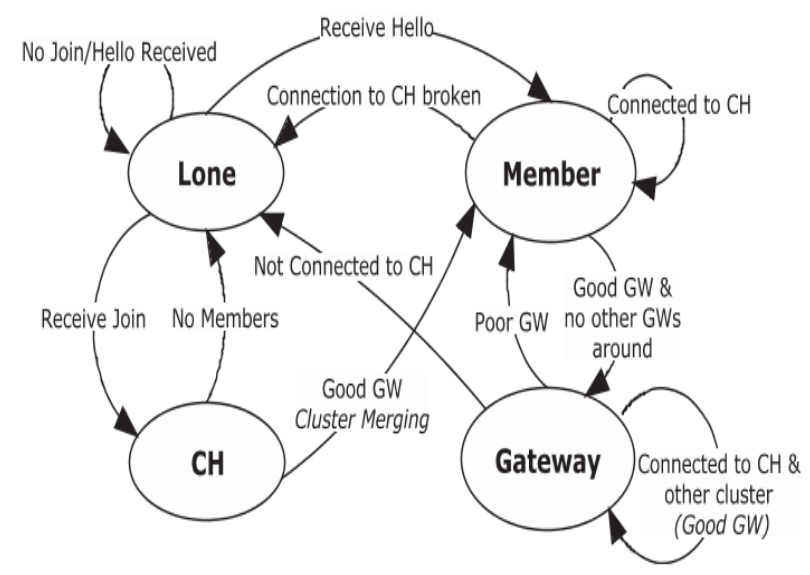

Figure 3. Clustering protocol flow of message.

\subsection{Certificate based Schemes}

This protocol uses clustering scheme for certificate generations and also revocations approaches are classified as certificate based schemes. Some of the protocols which fall in this category are as follows:.

Tahani Gazdar et al. $\stackrel{14}{ }$ discussed dynamic public's key infrastructures for VANET that aim for the distribution the roles of central certifications authority's among the set of dynamic chosen Certificate Authorities (CA)s. The elections of dynamic CA are based on clustering algorithms in which cluster head performs the role of certificates authority.

In Qingwei Zhang et al. $\frac{15}{{ }^{-}}$introduced a certificate revocations status validation schemes. The certificate validations are more time sensitive as every vehicle receives large numbers of message in least amount of time. This scheme employs a k-means clustering techniques to boost up the efficiencies of certificate validations. Thus, it enhance the security's of VANET.

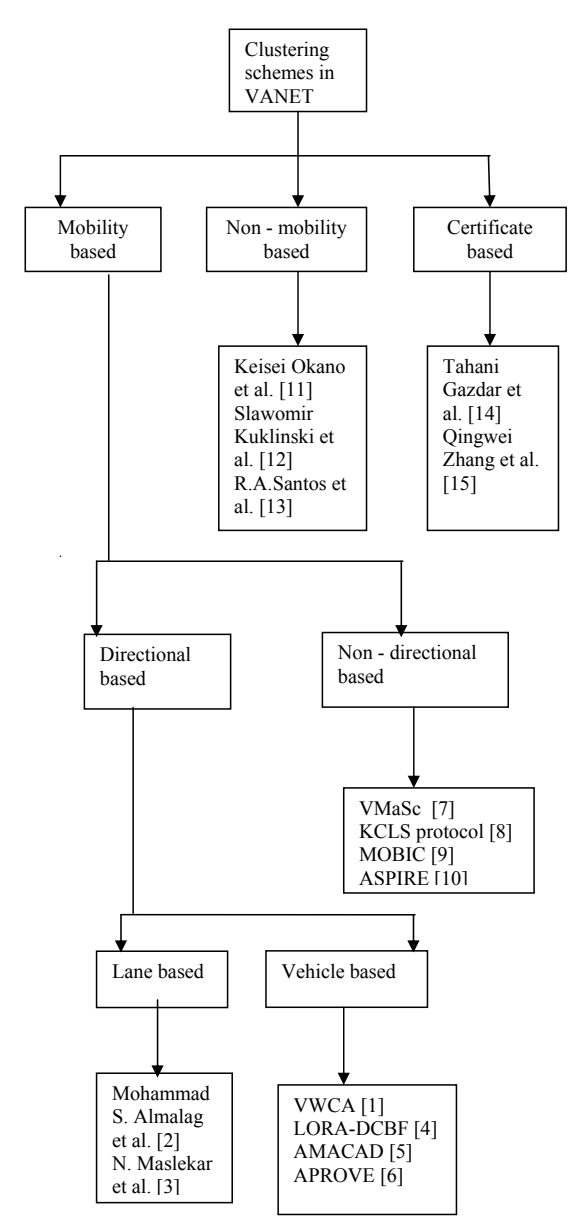

Figure 4. Classification of clustering schemes. 


\section{Conclusion}

The customary grouping's calculation created for versatile impromptu system is hard to actualize for VANET as the hub in this systems are very portable. Likewise, the hubs of vehicular impromptu system don't act power challenge like postured by hub of portable specially appointed system. Subsequently, new bouncing's calculation are proposed and produced for vehicular specially appointed system which go for steady and compelling groups in the system. Vehicular specially appointed system conventions of grouping plans are arranged into three classes as portability based bunching plans, nonversatility based grouping plans and endorsement based bunching plans. This kind of arrangement depends on the parameters used to overview on grouping systems utilized as a part of VANET.

The initial two classes of versatility of hubs is the principle parameter for arrangement. The versatility based grouping plan can be further arrange as in light of course of vehicle in particular heading based bunching plans and non-bearing based bunching plans. The course based grouping plans utilizes bearing of the vehicle or movement as one of its parameter for bunch development. Non-heading based grouping plan utilizes parameter other than course. The course based bunching calculation is further ordered into the path based grouping plans and vehicle based grouping plans. The path based grouping plan utilizes the course of movement as one of its bunching parameters in which vehicle based grouping plan utilizes the bearing of vehicles as one of its grouping parameters. Conventions delegated non-bearing based bunching plan utilizes speed contrasts and thickness based metric as the grouping parameter. The testament based bunching calculation utilizes grouping methods for authentication era and repudiation in the VANET.

\section{Future work}

Although a large portion of the scientists is concentrating on the versatility of bunching convention of Vehicular Ad-hoc Networks. Still the transmission of message among the hubs is to be velocity up. Further upgrades can be made in vehicular systems to make it more effective.

\section{References}

1. Daeinabi A, Rahbar AG, Khademzadeh A.VWCA: An efficient clustering algorithm in vehicular adhoc networks. J Netw Comput Appl.2011; 34(1): 207-22..

2. Mohammad S. Almalag, Michele C. Weigle. Using traffic flow for cluster formation in vehicular Ad-hoc networks. IEEE 35th Conference on Local Computer Networks (LCN).2010. p. 631-36.

3. Maslekar N, Boussedjra M, Mouza J, Labiod H. A stable clustering algorithm for efficiency applications in VANETs. 7th International Wireless Communications and Mobile Computing Conference (IWCMC).2011. p. 1188-193.

4. Sadaf Momeni, Mahmood Fathy and Nasser Mozayani. Vehicular Ad-Hoc networks clustering. In: proc. of Advanced Satellite Mobile Systems.2008. p. 299-304.

5. Mildred M. Caballeros Morales, Choong Seon Hong and Young-Cheol Bang. An adaptable mobility-aware clustering algorithm in vehicular networks. In: proc. of Network Operations and Management Symposium (APNOMS).2011. p. 1-6.

6. Christine Shea, Behnam Hassanabadi, Shahrokh Valaee. Mobility-based clustering in VANETs using affinity propagation. In: proc. of Global Telecommunications Conference. 2009. p. 1-6.

7. Seyhan Ucar, Sinem Coleri Ergen, Oznur Ozkasap. VMaSC: Vehicular multi-hop algorithm for stable clustering in vehicular Ad Hoc networks. In: proc. of Wireless Communications and Networking Conference (WCNC). 2013. p. 2381-86.

8. Zhang L, Elsayed $\mathrm{H}$ and Barka E. A novel location service protocol in multi-hop clustering vehicular Ad Hoc networks. In: proc. of International Conference on Innovations in Information Technology (IIT).2011. p. 386-91.

9. Basu P.A mobility based metric for clustering in mobile Ad Hoc networks. International Conference on Distributed Computing Systems Workshop. 2011. p. 413-18.

10. Agop Koulakezian.ASPIRE: Adaptive service provider infrastructure for VANETs. The University of Toronto. 2011.

11. Okano K, Ohta T, Kakuda Y. A dynamic network gateway selection scheme based on autonomous clustering for heterogeneous mobile Ad Hoc network environment. In: proceedings of IEEE Globecom Workshops (GC Wkshps). 2012. p. 513-17.

12. Kuklinski S, Wolny G. Density based clustering algorithm for VANETs. 5th International Conference on Testbeds and Research Infrastructures for the Development of Networks and Communities and Workshops. 2009. p. 1-6.

13. Santos RER, Seed N. Inter vehicular data exchange between fast moving road traffic using ad-hoc cluster based location 
algorithm and $802.11 \mathrm{~b}$ direct sequence spread spectrum radio. PostGraduate Networking Conference. 2003.

14. Gazdar T, Belghith A, Benslimane A. A cluster based secure architecture for vehicular ad hoc networks. IEEE/ ACS International Conference on Computer Systems and Applications (AICCSA). 2010. p. 1-8.
15. Zhang Q, Almulla M, Ren Y, Boukerche A. An efficient certificate revocation validation scheme with $\mathrm{k}$-Means clustering for vehicular Ad hoc networks. IEEE Symposium on Computers and Communications (ISCC). 2012 p. 862-67. 